\title{
Maternal Biomarkers of Acetaminophen Use and Offspring Attention Deficit Hyperactivity Disorder
}

Yuelong Ji ${ }^{1}$, Anne W. Riley ${ }^{1}$, Li-Ching Lee ${ }^{2,3}$, Xiumei Hong ${ }^{1}$, Guoying Wang ${ }^{1}$, Hui-Ju Tsai ${ }^{4}$, Noel T. Mueller ${ }^{2}$, Colleen Pearson ${ }^{5}$, Jessica Thermitus ${ }^{5}$, Anita Panjwani ${ }^{6}$, Hongkai Ji ${ }^{7}$, Tami R. Bartell ${ }^{8}$, Irina Burd ${ }^{9}$, M. Daniele Fallin ${ }^{2,3}$ and Xiaobin Wang 1,10,*

1 Center on the Early Life Origins of Disease, Department of Population, Family and Reproductive Health, Johns Hopkins University Bloomberg School of Public Health, 615 N Wolfe St, Baltimore, MD 21205, USA; yji7@jhu.edu (Y.J.); ariley1@jhu.edu (A.R.); xhong3@jhu.edu (X.H.); gwang24@jhu.edu (G.W.)

2 Department of Epidemiology, Johns Hopkins University Bloomberg School of Public Health, 615 N Wolfe St, Baltimore, MD 21205, USA; llee38@jhu.edu (L.-C.L.); noeltmueller@jhu.edu (N.T.M.); dfallin@jhu.edu (M.D.F.)

3 Wendy Klag Center for Autism and Developmental Disabilities \& Department of Mental Health, 615 N Wolfe St, Baltimore, MD 21205, USA

4 Division of Biostatistics and Bioinformatics, Institute of Population Health Sciences, National Health Research Institutes, Zhunan 35053, Taiwan; tsaihj@nhri.org.tw

5 Department of Pediatrics, Boston University School of Medicine and Boston Medical Center, 1 Boston Medical Center Pl, Boston, MA 02118, USA; Colleen.Pearson@bmc.org (C.P.); Jessica.Thermitus@bmc.org (J.T.)

6 Department of International Health, Johns Hopkins University Bloomberg School of Public Health, 615 N Wolfe St, Baltimore, MD 21205, USA; apanjwani@jhu.edu

7 Department of Biostatistics, Johns Hopkins University Bloomberg School of Public Health, 615 N Wolfe St, Baltimore, MD 21205, USA; hji@jhu.edu

8 Stanley Manne Children's Research Institute, Mary Ann \& J. Milburn Smith Child Health Research, Outreach and Advocacy Center, Ann \& Robert H. Lurie Children's Hospital of Chicago, 2430 N Halsted St, Chicago, IL 60614, USA; TBartell@luriechildrens.org

9 Integrated Research Center for Fetal Medicine, Department of Gynecology and Obstetrics, Johns Hopkins University School of Medicine, 1800 Orleans St, Baltimore, MD 21287, USA; iburd@jhmi.edu

10 Division of General Pediatrics \& Adolescent Medicine, Department of Pediatrics, Johns Hopkins University School of Medicine, 1800 Orleans St, Baltimore, MD 21287, USA

* Correspondence: xwang82@jhu.edu; Tel.: +1-410-955-5824; Fax: +1-410-502-5831

Received: 9 June 2018; Accepted: 30 June 2018; Published: 3 July 2018

\begin{abstract}
Previous studies have suggested a positive association between self-reported maternal acetaminophen use during pregnancy and risk of attention deficit hyperactivity disorder (ADHD) in offspring. We sought to examine the prospective association between maternal plasma biomarkers of acetaminophen intake and ADHD diagnosis in the offspring. This report analyzed 1180 children enrolled at birth and followed prospectively as part of the Boston Birth Cohort, including 188 with ADHD diagnosis based on electronic medical record review. Maternal biomarkers of acetaminophen intake were measured in plasma samples obtained within 1-3 days postpartum. Odds ratios for having ADHD diagnosis or other developmental disorders were estimated using multinomial logistic regression models, adjusting for pertinent covariables. Compared to neurotypical children, we observed significant positive dose-responsive associations with ADHD diagnosis for each maternal acetaminophen biomarker. These dose-responsive associations persisted after adjusting for indication of acetaminophen use and other pertinent covariates; and were specific to ADHD, rather than other neurodevelopmental disorders. In the stratified analyses, differential point estimates of the associations were observed across some strata of covariates. However, these differences were not statistically significant. Maternal acetaminophen biomarkers were specifically associated with
\end{abstract}


increased risk of ADHD diagnosis in offspring. Additional clinical and mechanistic investigations are warranted.

Keywords: ADHD; acetaminophen; pregnancy

\section{Introduction}

Attention deficit hyperactivity disorder (ADHD) is one of the most common lifelong neurodevelopmental disorders in the world. Its prevalence among children ages 4-17 years in the U.S. increased significantly from $7.0 \%$ to $10.2 \%$ during the past two decades $[1,2]$. The rapid rise of ADHD cannot be attributed to genetic mutations. Indeed, multiple social and environmental risk factors have been associated with the development of ADHD, including family-related factors [3-15], maternal obesity [16,17], maternal smoking [8,18-20], maternal drinking [8,21], low birthweight and preterm birth [22], exposure to organophosphates [23], polychlorinated biphenyls [24,25], and lead exposure [24,26-29]. These findings underscore the role of environmental factors in the etiology of ADHD, and the need to explore other important yet unknown risk factors for ADHD [30]. Acetaminophen is widely used and recommended over-the-counter medication for fever and pain relief during pregnancy. The extent of acetaminophen use during pregnancy is over $65 \%$ in the U.S. and over 50\% in Europe [31,32]. The inhibition of prostaglandin synthesis is part of the therapeutic effect of acetaminophen [33]. Prostaglandins not only act as fever determinants but also play essential roles in brain function, including long-term potentiation [34], learning [35], and cerebellar development [33]. Because of its widespread use and role in brain function, the potential unknown adverse effects of acetaminophen use on developing fetal brain need to be clarified [36].

Since 2013, research studies analyzing five prospective cohorts from Europe and New Zealand have consistently shown a positive association between maternal intake of acetaminophen during pregnancy and increased risk of ADHD and its related symptoms in offspring [37-41]. The Society for Maternal-Fetal Medicine and the Food and Drug Administration expressed concern that the data from these recent studies are still too inconclusive to draw any causal inference between prenatal acetaminophen use and ADHD development in offspring [42,43]. Their primary criticisms included the use of self-reported exposure, lack of dose quantification, and unmeasured confounders [42]. To address the concerns and criticisms related to previous studies and improve our understanding of acetaminophen's effect during pregnancy and beyond, there is a need for a well-designed prospective birth cohort study with blood samples available to measure maternal acetaminophen levels. Currently, no such study exists.

In this study, using the data from the Boston Birth Cohort, we sought to examine the prospective association between maternal plasma acetaminophen metabolites levels measured within a few days after delivery and ADHD diagnosis in the offspring. Given the timing of our biomarker measurement, we are poised to address a specific question that has not been explored in the previous study: Is the maternal use of acetaminophen during peripartum period (as reflected in plasma biomarker levels) associated with increased risk of ADHD diagnosis in offspring?

\section{Methods}

\subsection{Sample}

Initiated in 1998, mother/infant pairs were recruited at birth from the Boston Medical Center (BMC) for participation in the Boston Birth Cohort (BBC) [44,45]. The BMC serves a predominately low income, urban, minority population and is also the largest safety net hospital in New England. Eligible mothers were those who delivered a singleton live birth at BMC. They were approached for consent and enrollment within 24-72 h after delivery. Infants who continued to receive pediatric primary or 
specialty care at BMC were invited (beginning at age six months) to participate in the follow-up study in which they are prospectively followed from birth onwards [44,46,47]. After obtaining informed consent, a standardized questionnaire was administered by trained research staff, and a maternal venous blood sample was obtained. Mothers who conceived via in vitro fertilization, multiple-gestation pregnancies, deliveries induced by maternal trauma, and/or newborns with substantial congenital disabilities were not eligible for participation. Both the baseline study and the follow-up study have been approved by the Institutional Review Boards (IRB) of Boston University Medical Center and Johns Hopkins Bloomberg School of Public Health.

As illustrated in the study flowchart, of the 3098 children in the postnatal follow-up study in the BBC, 1412 mothers had sufficient plasma samples for metabolomic assay. Of the mothers with metabolomic data, we further excluded 232 participants who had missing data for key covariates. Our final sample comprised 1180 mother/infant pairs with all pertinent data: exposure, outcome, and covariates (Figure S1). Although this is a subset of the BBC, this sample was similar to the excluded sample in terms of baseline maternal and newborn characteristics (Table S1), except for having a slightly higher percentage of black children, longer gestation, and higher birthweight.

\subsection{Definitions for $A D H D, A S D$, Other $D D$, and Neurotypical Children}

We extracted information regarding each child's neuro-developmental diagnoses as documented in their electronic medical records (EMRs). Beginning in 2003, BMC implemented EMR as part of routine data collection for both well-child and specialty clinical visits. The primary and secondary diagnoses for each clinical visit were coded in the EMR using the International Classification of Diseases, Ninth Revision (ICD-9) (before 1 October 2015) and ICD-10 (after 1 October 2015). Thus, all children in the BBC postnatal follow-up study with a related ICD-9 (314.0, 314.00, 314.01, 314.1, 314.2, 314.8, or 314.9) or ICD-10 (F90.0, F90.1, F90.2, F90.8, or F90.9) code included in their EMR between 2003 and 2016 were classified as having ADHD. Similarly, children with an ICD-9 (299.0, 299.00, 299.01, 299.8, 299.80, 299.81, 299.9, 299.90, or 299.91) or ICD-10 (F84.0, F84.8, or F84.9) code were classified as having an autism spectrum disorder (ASD). Furthermore, children with any of following developmental disorder diagnoses noted in their EMR were classified as having other developmental disorders (other DD): developmental delays, or intellectual disabilities. Children without any diagnosis of ASD, ADHD, developmental delays, or intellectual disabilities were classified as neurotypical (NT). Table S2 lists the ICD-9 and ICD-10 codes for each developmental disorder diagnosis.

\subsection{Maternal Biomarkers of Acetaminophen Use}

Maternal plasma biomarkers of acetaminophen use were measured using nonfasting blood samples obtained within 1-3 days postpartum. As illustrated in Figure 1, the main metabolites (and proportion) of acetaminophen include unchanged acetaminophen ( 5\%), acetaminophen glucuronide (52-57\%), acetaminophen sulfate (30-44\%), and hepatotoxic N-acetyl-p-benzoquinone imine (NAPQI) (5-10\%). NAPQI can be further detoxified as 3-(N-Acetyl-L-cystein-S-yl) acetaminophen [48]. The peak intensity of unchanged acetaminophen, acetaminophen glucuronide, and 3-(N-Acetyl-L-cystein-S-yl) acetaminophen in maternal blood was measured using liquid chromatography-tandem mass spectrometry (LC-MS) techniques at the MIT Broad Institute Metabolite Profiling Laboratory. All the intensity levels were inverse normal transformed for the subsequent statistical analyses.

\subsection{Covariates}

Based on previous literature $[29,37-41]$, the following covariates were included as potential confounders: maternal age at delivery, maternal race/ethnicity, maternal education, smoking from six months before pregnancy to birth (never smoked, quit during this period, continued to smoke during this period), ever drank alcohol from six months before pregnancy to birth, maternal pre-pregnancy BMI, parity, maternal fever during pregnancy, intrauterine infection/inflammation, baby's sex, delivery type, gestational age, birthweight, breastfeeding, early childhood lead levels, and maternal 
high-density lipoprotein (HDL) levels. Maternal demographic covariates were collected using a standard questionnaire interview. Maternal and child clinically-related covariates were abstracted from their medical records, respectively. The lead levels of the children were collected as part of the pediatric routine lead screening and extracted from their EMRs. The first lead levels measured were chosen for the analysis. Maternal plasma HDL levels were measured using nonfasting blood samples obtained within 1-3 days postpartum.

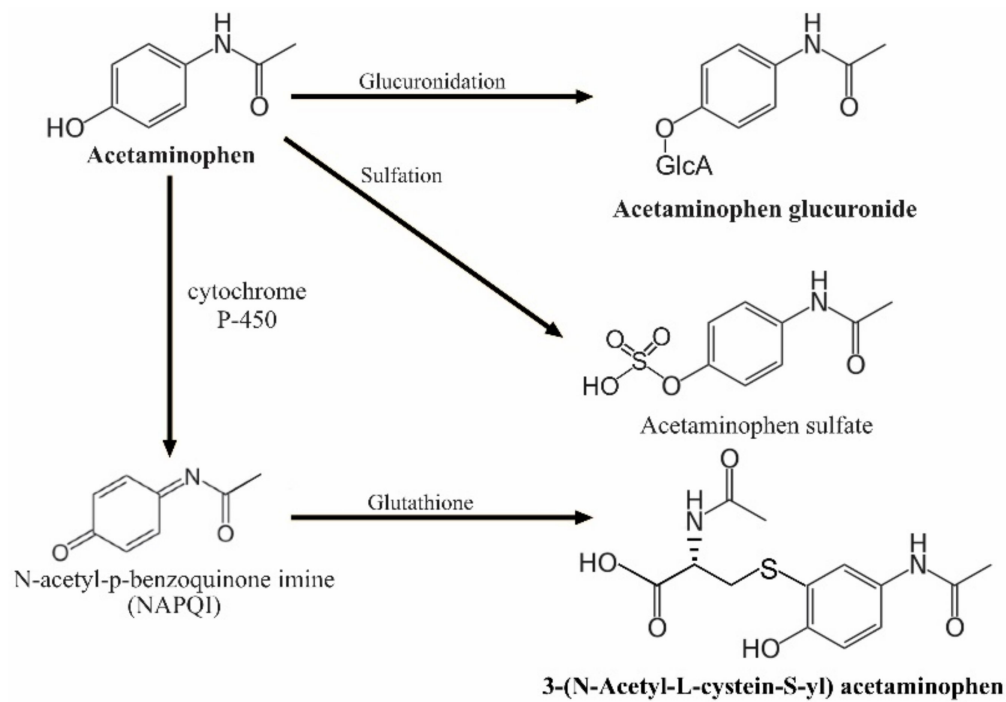

Figure 1. Pathways of acetaminophen metabolism (Bolded metabolites were measured in this study).

\subsection{Statistical Analyses}

The characteristics of the study sample for the ADHD, ASD (excluding participants with ADHD diagnosis), other DD, and NT groups were compared using one-way ANOVA for continuous variables and $\chi^{2}$ tests for categorical variables. The main exposures analyzed in this study were maternal acetaminophen metabolite levels, which were inverse normal transformed to approximate the normal distribution. The inverse normal transformed unchanged acetaminophen levels were also categorized into tertiles. Due to the high rate of non-detection, the inverse normal transformed acetaminophen glucuronide and 3-(N-Acetyl-L-cystein-S-yl) acetaminophen levels were categorized into three groups: no detection, below median, above median of detected values. Based on previous findings regarding the proportions of acetaminophen metabolites typically found in blood samples [48], we further calculated a variable to reflect overall "acetaminophen burden" by combining all of the acetaminophen metabolites levels with a weighting of their proportions in the acetaminophen metabolic pathway (acetaminophen burden = (unchanged acetaminophen $/ 5 \%+$ acetaminophen glucuronide/50\%+ 3-(N-Acetyl-L-cystein-S-yl) acetaminophen /5\%)/60\%) [48]. The acetaminophen burden levels were then also categorized into three groups: no detection, below median, and above median. Each child's early life lead level was converted into a binary variable ( $5 \mu \mathrm{g} / \mathrm{dL}$ as the cutoff) for analysis based on CDC guidelines [49]. Maternal HDL level was cut at $60 \mathrm{mg} / \mathrm{dL}$ for analyses based on previous finding [50].

We conducted sequential multinomial logistic regression models to examine the association between maternal acetaminophen metabolite levels and the risk of having ADHD diagnosis, ASD diagnosis (excluding ADHD diagnosis), or other DD diagnosis in offspring. Multinomial logistic regression is a method that generalizes logistic regression to the tests with more than two possible discrete outcomes [51]. Except for the fact that dependent variable is categorical rather than binary, the basic setup is similar to logistic regression [51]. Models included a crude (unadjusted) model (Model 1); a model adjusted for maternal age at delivery, maternal race/ethnicity, maternal education, smoking during pregnancy, drinking during pregnancy, parity, maternal pre-pregnancy 
BMI, baby's sex, delivery type, gestational age, and birthweight (Model 2); and models further adjusted for maternal fever during pregnancy (Model 3), intrauterine infection/inflammation (Model 4), and breastfeeding (Model 5), separately, and combined (Model 6). We also performed stratified analyses by each stratum of covariates (including child's early life lead levels [52] and maternal HDL levels [50]) for binary acetaminophen burden (detected vs. no detection) using univariate logistic regression comparing those with an ADHD diagnosis to the NT group. For the sensitivity analyses, we repeated the sequential models using propensity score weighted multinomial logistic regression. The propensity score was calculated based on all the covariates in Model 6 using psmatch 2 package. We further repeated the sequential models for each of the following outcomes: "ADHD only" (excluding ASD diagnosis), "ASD only" (excluding ADHD diagnosis), and "ADHD and ASD" (having both diagnoses), all compared to the NT group. STATA ${ }^{\circledR}$ version 14.0 software was used to perform all analyses (Stata Corporation, College Station, TX, USA).

\section{Results}

In the final sample, there were 188 children with a diagnosis of ADHD, 44 children with a diagnosis of ASD (without ADHD diagnosis), 344 children with a diagnosis of other DD, and 604 NT children. The median age at first ADHD diagnosis was 7 years. Figure 2 shows the distribution of each acetaminophen metabolite and acetaminophen burden across diagnosis groups. Both the ADHD diagnosis and ASD diagnosis (without ADHD diagnosis) groups had more mothers with higher levels of acetaminophen metabolites compared to the NT and other DD diagnosis groups. Table 1 presents the crude comparisons of maternal and child characteristics among the ADHD diagnosis, ASD diagnosis (without ADHD diagnosis), other DD diagnosis, and NT groups. The ADHD and ASD groups had the highest percentage of detectable unchanged acetaminophen and its metabolites. Mothers of children with any ADHD diagnosis were also more likely to have below college degree education, ever smoked before or during pregnancy, and C-section delivery, compared with the NT group. Children with any ADHD, ASD, or any other DD diagnosis were more likely to be male, born prematurely and have had low birthweight, compared with the NT group.
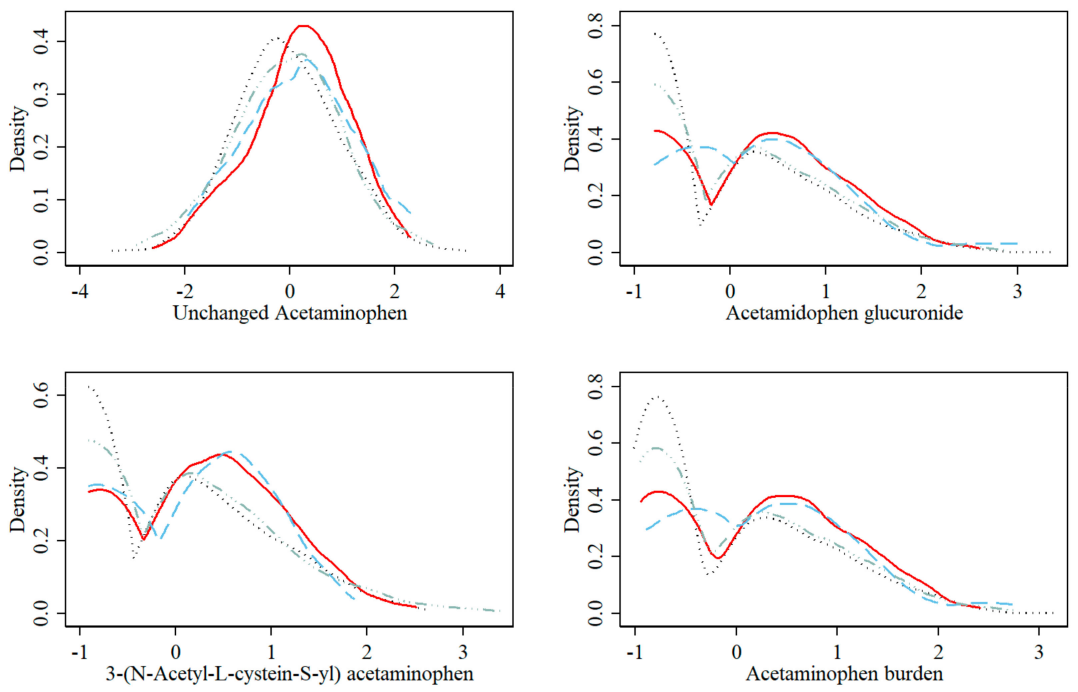

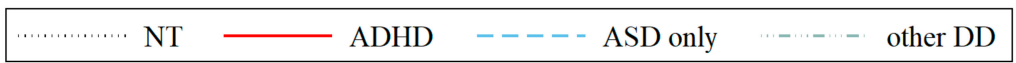

Figure 2. Comparison of the distributions of acetaminophen metabolites and acetaminophen burden by specific diagnosis groups. NT was defined as free of any developmental disorder diagnosis; ADHD was defined as any ADHD diagnosis; ASD only was defined as any ASD diagnosis without having an ADHD diagnosis; other DD was defined as any developmental disorder diagnosis other than ASD and ADHD. 
Table 1. Maternal and child characteristics for children with ADHD diagnosis, ASD diagnosis (excluding ADHD), other developmental disorder diagnosis (other DD), and neurotypical children (NT).

\begin{tabular}{|c|c|c|c|c|c|c|}
\hline Variable & Total, $N(\%)$ & $\mathrm{NT}, N(\%)$ & ADHD, $N(\%)$ & ASD, $N(\%)$ & Other DD, $N(\%)$ & $p$-Value $\ddagger$ \\
\hline Total & $1180(100)$ & $604(51.19)$ & $188(15.93)$ & $44(3.73)$ & $344(29.15)$ & \\
\hline Maternal Age & & & & & & 0.101 \\
\hline$<35$ & $965(81.78)$ & $510(84.44)$ & $151(80.32)$ & $34(77.27)$ & $270(78.49)$ & \\
\hline$\geq 35$ & $215(18.22)$ & $94(15.56)$ & $37(19.68)$ & $10(22.73)$ & $74(21.51)$ & \\
\hline Maternal race/ethnicity & & & & & & 0.073 \\
\hline Black & $809(68.56)$ & $425(70.36)$ & $126(67.02)$ & $26(59.09)$ & $232(67.44)$ & \\
\hline White & $48(4.07)$ & $24(3.97)$ & $11(5.85)$ & $2(4.55)$ & $11(3.20)$ & \\
\hline Hispanic & $256(21.69)$ & $112(18.54)$ & $44(23.40)$ & 15 (34.09) & 85 (24.71) & \\
\hline Others & $67(5.68)$ & $43(7.12)$ & $7(3.72)$ & $1(2.27)$ & $16(4.65)$ & \\
\hline Education level & & & & & & 0.208 \\
\hline Below college degree & $1033(87.54)$ & $520(86.09)$ & $172(91.49)$ & $37(84.09)$ & $304(88.37)$ & \\
\hline College degree or above & $147(12.46)$ & $84(13.91)$ & $16(8.51)$ & $7(15.91)$ & $40(11.63)$ & \\
\hline \multicolumn{2}{|c|}{ Smoking before or during pregnancy } & & & & & 0.018 \\
\hline Never & $977(82.80)$ & $520(86.09)$ & $141(75.00)$ & $38(86.36)$ & $278(80.81)$ & \\
\hline Quitter & $90(7.63)$ & $38(6.29)$ & $18(9.57)$ & $3(6.82)$ & $31(9.01)$ & \\
\hline Continuous & $113(9.58)$ & $46(7.62)$ & $29(15.43)$ & $3(6.82)$ & 35 (10.17) & \\
\hline \multicolumn{2}{|c|}{ Drinking before or during pregnancy } & & & & & 0.491 \\
\hline No & $1086(92.03)$ & $560(92.72)$ & $173(92.02)$ & $38(86.36)$ & $315(91.57)$ & \\
\hline Yes & $94(7.97)$ & $44(7.28)$ & $15(7.98)$ & $6(13.64)$ & $29(8.43)$ & \\
\hline \multicolumn{2}{|l|}{ Parity } & & & & & 0.484 \\
\hline Nulliparous & $527(44.66)$ & $281(46.52)$ & $85(45.21)$ & $18(40.91)$ & $143(41.57)$ & \\
\hline Multiparous & $653(55.34)$ & $323(53.48)$ & $103(54.79)$ & $26(59.09)$ & $201(58.43)$ & \\
\hline \multicolumn{2}{|l|}{ Child's sex } & & & & & $<0.001$ \\
\hline Female & $576(48.81)$ & $351(58.11)$ & $49(26.06)$ & $14(31.82)$ & $162(47.09)$ & \\
\hline Male & $604(51.19)$ & $253(41.89)$ & $139(73.94)$ & $30(68.18)$ & $182(52.91)$ & \\
\hline \multicolumn{2}{|l|}{ Delivery type } & & & & & 0.008 \\
\hline C-section & $426(36.10)$ & $192(31.79)$ & $75(39.89)$ & $22(50.00)$ & $137(39.83)$ & \\
\hline Vaginal & $754(63.90)$ & $412(68.21)$ & $113(60.11)$ & $22(50.00)$ & $207(60.17)$ & \\
\hline \multicolumn{2}{|l|}{ Maternal fever } & & & & & 0.594 \\
\hline No & $1108(93.90)$ & $570(94.37)$ & $178(94.68)$ & $42(95.45)$ & $318(92.44)$ & \\
\hline Yes & $72(6.10)$ & $34(5.63)$ & $10(5.32)$ & $2(4.55)$ & $26(7.56)$ & \\
\hline \multicolumn{2}{|c|}{ Intrauterine infection/inflammation } & & & & & 0.136 \\
\hline No & $1023(86.69)$ & $537(88.91)$ & $157(83.51)$ & $38(86.36)$ & $291(84.59)$ & \\
\hline Yes & $157(13.31)$ & $67(11.09)$ & $31(16.49)$ & $6(13.64)$ & $53(15.41)$ & \\
\hline \multicolumn{2}{|l|}{ Maternal BMI } & & & & & 0.304 \\
\hline$<18.50$ & $41(3.47)$ & $20(3.31)$ & $9(4.79)$ & $2(4.55)$ & $10(2.91)$ & \\
\hline $18.50-24.99$ & $514(43.56)$ & $284(47.02)$ & $72(38.30)$ & $15(34.09)$ & $143(41.57)$ & \\
\hline $25-29.99$ & $337(28.56)$ & $164(27.15)$ & $58(30.85)$ & $11(25.00)$ & $104(30.23)$ & \\
\hline$>30$ & $288(24.41)$ & $136(22.52)$ & $49(26.06)$ & $16(36.36)$ & $87(25.29)$ & \\
\hline \multicolumn{2}{|l|}{ Breastfeeding } & & & & & 0.351 \\
\hline Bottle only & $286(24.24)$ & $142(23.51)$ & $55(29.26)$ & $9(20.45)$ & $80(23.26)$ & \\
\hline Both or breastfed only & $894(75.76)$ & $462(76.49)$ & $133(70.74)$ & $35(79.55)$ & $264(76.74)$ & \\
\hline \multicolumn{2}{|l|}{ Unchanged acetaminophen * } & & & & & 0.027 \\
\hline First tertile & $411(34.83)$ & $227(37.58)$ & $46(24.47)$ & $13(29.55)$ & $125(36.34)$ & \\
\hline Second tertile & 375 (31.78) & 192 (31.79) & 66 (35.11) & $12(27.27)$ & $105(30.52)$ & \\
\hline Third tertile & $394(33.39)$ & $185(30.63)$ & $76(40.43)$ & $19(43.18)$ & $114(33.14)$ & \\
\hline \multicolumn{2}{|c|}{ 3-(N-Acetyl-L-cystein-S-yl) acetaminophen * } & & & & & 0.013 \\
\hline No detection & $441(37.37)$ & $248(41.06)$ & $51(27.13)$ & $15(34.09)$ & $127(36.92)$ & \\
\hline Below median & $361(30.59)$ & $182(30.13)$ & $62(32.98)$ & $10(22.73)$ & $107(31.10)$ & \\
\hline Above median & $378(32.03)$ & $174(28.81)$ & $75(39.89)$ & $19(43.18)$ & $110(31.98)$ & \\
\hline \multicolumn{2}{|l|}{ Acetaminophen glucuronide * } & & & & & 0.018 \\
\hline No detection & $531(45.00)$ & $299(49.50)$ & $68(36.17)$ & $15(34.09)$ & $149(43.31)$ & \\
\hline Below median & $315(26.69)$ & $152(25.17)$ & $52(27.66)$ & $15(34.09)$ & $96(27.91)$ & \\
\hline Above median & $334(28.31)$ & $153(25.33)$ & $68(36.17)$ & $14(31.82)$ & $99(28.78)$ & \\
\hline \multicolumn{2}{|l|}{ Acetaminophen burden ${ }^{* *}$} & & & & & 0.027 \\
\hline No detection & $531(45.00)$ & $299(49.50)$ & $68(36.17)$ & $15(34.09)$ & $149(43.31)$ & \\
\hline Below median & $315(26.69)$ & $151(25.00)$ & $54(28.72)$ & $14(31.82)$ & $96(27.91)$ & \\
\hline Above median & $334(28.31)$ & $154(25.50)$ & $66(35.11)$ & $15(34.09)$ & $99(28.78)$ & \\
\hline \multicolumn{2}{|l|}{ Gestational age, week } & & & & & $<0.001$ \\
\hline Mean (SD) & $37.9(3.3)$ & $38.5(2.5)$ & $37.3(3.6)$ & $37.0(4.6)$ & $37.2(4.0)$ & \\
\hline Birthweight, g & & & & & & $<0.001$ \\
\hline Mean (SD) & $\begin{array}{l}2966.2 \\
(789.9)\end{array}$ & $\begin{array}{l}3085.5 \\
(669.7)\end{array}$ & $2865.0(819.4)$ & $\begin{array}{c}2860.9 \\
(1026.2)\end{array}$ & $2825.5(898.0)$ & \\
\hline
\end{tabular}

Note: NT was defined as free of any developmental disorder diagnosis; ADHD was defined as any ADHD diagnosis; ASD was defined as any ASD diagnosis without having an ADHD diagnosis; other DD was defined as any developmental disorder diagnosis other than ASD and ADHD. ₹ The $p$-values were obtained from $\chi^{2}$ tests or one-way ANOVA among the four diagnosis groups; ${ }^{*}$ Inverse normal transformed intensity; ${ }^{* *}$ Sum of all the acetaminophen metabolites. 
Table 2 shows the sequential multinomial logistic regression model results for the relationship between acetaminophen metabolites and the risk of ADHD diagnosis, ASD diagnosis (excluding ADHD), or other DD diagnosis, and before and after adjusting for pertinent covariates. The group with the highest plasma level of each acetaminophen metabolite was significantly associated with the risk of ADHD diagnosis, and the effect size was similar across all models. Moreover, we identified dose-responsive patterns across all acetaminophen metabolites and burden. Compared to levels in the non-detection category, below median and above median levels of maternal acetaminophen burden were associated with a $58 \%$ and $88 \%$ increase in the odds of ADHD diagnosis, respectively (Model 6: OR for below median $=1.58,95 \%$ CI $(1.02,2.46)$; OR for above median $=1.88,95 \%$ CI $(1.18,3.00)$ ). In contrast, the risks of ASD diagnosis and other DD diagnoses were not significantly associated with maternal plasma levels of acetaminophen metabolites across all models. Table S3 further confirms that in our sensitivity analyses the acetaminophen metabolite levels were specifically associated with the risk of having an ADHD diagnosis (without ASD diagnosis). Table S4 presents the results of propensity score weighted multinomial logistic regression models, which is an additional method of controlling for unmeasured confounders. It also shows similar findings to Table 2.

Table 2. The association between maternal acetaminophen metabolites and the risk of ADHD diagnosis, ASD diagnosis (excluding ADHD), and other DD diagnosis in offspring.

\begin{tabular}{|c|c|c|c|c|c|c|c|c|c|c|}
\hline \multirow{2}{*}{\multicolumn{2}{|c|}{ Model }} & \multicolumn{3}{|c|}{ ADHD, 188 (15.9\%) } & \multicolumn{3}{|c|}{ ASD, 44 (3.7\%) } & \multicolumn{3}{|c|}{ Other DD, 344 (29.2\%) } \\
\hline & & Odds Ratio & $95 \%$ CI & $p$-Value & Odds Ratio & $95 \% \mathrm{CI}$ & $p$-Value & Odds Ratio & $95 \% \mathrm{CI}$ & $p$-Value \\
\hline \multicolumn{11}{|c|}{ Unchanged acetaminophen* } \\
\hline \multirow{2}{*}{ Model 1} & Second tertile & 1.70 & $(1.11,2.59)$ & 0.014 & 1.09 & $(0.49,2.45)$ & 0.832 & 0.99 & $(0.72,1.37)$ & 0.967 \\
\hline & Third tertile & 2.03 & $(1.34,3.07)$ & 0.001 & 1.79 & $(0.86,3.73)$ & 0.118 & 1.12 & $(0.81,1.54)$ & 0.490 \\
\hline \multirow[t]{2}{*}{ Model 2} & Second tertile & 1.72 & $(1.10,2.70)$ & 0.018 & 0.98 & $(0.43,2.27)$ & 0.970 & 0.99 & $(0.71,1.40)$ & 0.977 \\
\hline & Third tertile & 2.08 & $(1.29,3.35)$ & 0.003 & 1.38 & $(0.60,3.18)$ & 0.451 & 0.94 & $(0.65,1.35)$ & 0.732 \\
\hline \multirow{2}{*}{ Model 3} & Second tertile & 1.73 & $(1.10,2.72)$ & 0.017 & 0.99 & $(0.43,2.30)$ & 0.989 & 0.97 & $(0.69,1.37)$ & 0.883 \\
\hline & Third tertile & 2.08 & $(1.29,3.35)$ & 0.003 & 1.39 & $(0.60,3.20)$ & 0.443 & 0.93 & $(0.65,1.35)$ & 0.706 \\
\hline \multirow[t]{2}{*}{ Model 4} & Second tertile & 1.71 & $(1.09,2.68)$ & 0.020 & 0.98 & $(0.42,2.27)$ & 0.968 & 0.98 & $(0.70,1.39)$ & 0.931 \\
\hline & Third tertile & 2.06 & $(1.28,3.33)$ & 0.003 & 1.38 & $(0.60,3.18)$ & 0.453 & 0.93 & $(0.65,1.35)$ & 0.705 \\
\hline \multirow[t]{2}{*}{ Model 5} & Second tertile & 1.72 & $(1.10,2.70)$ & 0.018 & 0.98 & $(0.42,2.26)$ & 0.961 & 0.99 & $(0.70,1.39)$ & 0.958 \\
\hline & Third tertile & 2.06 & $(1.28,3.32)$ & 0.003 & 1.40 & $(0.61,3.23)$ & 0.432 & 0.94 & $(0.65,1.36)$ & 0.749 \\
\hline \multirow[t]{2}{*}{ Model 6} & Second tertile & 1.74 & $(1.10,2.73)$ & 0.017 & 0.99 & $(0.43,2.29)$ & 0.979 & 0.97 & $(0.69,1.37)$ & 0.869 \\
\hline & Third tertile & 2.05 & $(1.27,3.32)$ & 0.003 & 1.40 & $(0.60,3.24)$ & 0.433 & 0.93 & $(0.65,1.35)$ & 0.718 \\
\hline \multicolumn{11}{|c|}{ 3-(N-Acetyl-L-cystein-S-yl) acetaminophen * } \\
\hline \multirow[t]{2}{*}{ Model 1} & Below median & 1.66 & $(1.09,2.51)$ & 0.018 & 0.91 & $(0.40,2.07)$ & 0.819 & 1.15 & $(0.83,1.58)$ & 0.399 \\
\hline & Above median & 2.10 & $(1.40,3.14)$ & $<0.001$ & 1.81 & $(0.89,3.65)$ & 0.100 & 1.23 & $(0.90,1.70)$ & 0.198 \\
\hline \multirow[t]{2}{*}{ Model 2} & Below median & 1.68 & $(1.08,2.61)$ & 0.021 & 0.73 & $(0.31,1.72)$ & 0.474 & 1.08 & $(0.77,1.52)$ & 0.644 \\
\hline & Above median & 2.06 & $(1.28,3.31)$ & 0.003 & 1.21 & $(0.53,2.75)$ & 0.653 & 0.96 & $(0.66,1.40)$ & 0.835 \\
\hline \multirow[t]{2}{*}{ Model 3} & Below median & 1.70 & $(1.09,2.65)$ & 0.020 & 0.74 & $(0.31,1.75)$ & 0.494 & 1.05 & $(0.75,1.48)$ & 0.763 \\
\hline & Above median & 2.06 & $(1.28,3.31)$ & 0.003 & 1.22 & $(0.53,2.78)$ & 0.640 & 0.95 & $(0.65,1.38)$ & 0.789 \\
\hline \multirow[t]{2}{*}{ Model 4} & Below median & 1.66 & $(1.06,2.58)$ & 0.025 & 0.73 & $(0.31,1.72)$ & 0.468 & 1.06 & $(0.76,1.49)$ & 0.716 \\
\hline & Above median & 2.04 & $(1.27,3.28)$ & 0.003 & 1.20 & $(0.53,2.75)$ & 0.661 & 0.95 & $(0.65,1.38)$ & 0.785 \\
\hline \multirow[t]{2}{*}{ Model 5} & Below median & 1.67 & $(1.07,2.60)$ & 0.024 & 0.74 & $(0.31,1.75)$ & 0.497 & 1.09 & $(0.78,1.53)$ & 0.619 \\
\hline & Above median & 2.04 & $(1.27,3.28)$ & 0.003 & 1.23 & $(0.54,2.82)$ & 0.621 & 0.97 & $(0.67,1.41)$ & 0.868 \\
\hline \multirow[t]{2}{*}{ Model 6} & Below median & 1.68 & $(1.08,2.63)$ & 0.022 & 0.75 & $(0.32,1.78)$ & 0.513 & 1.06 & $(0.75,1.49)$ & 0.734 \\
\hline & Above median & 2.03 & $(1.26,3.27)$ & 0.004 & 1.23 & $(0.54,2.82)$ & 0.626 & 0.96 & $(0.66,1.39)$ & 0.811 \\
\hline \multicolumn{11}{|c|}{ Acetaminophen glucuronide* } \\
\hline \multirow[t]{2}{*}{ Model 1} & Below median & 1.50 & $(1.00,2.27)$ & 0.051 & 1.97 & $(0.94,4.13)$ & 0.074 & 1.27 & $(0.92,1.75)$ & 0.150 \\
\hline & Above median & 1.95 & $(1.33,2.88)$ & 0.001 & 1.82 & $(0.86,3.88)$ & 0.118 & 1.30 & $(0.94,1.79)$ & 0.110 \\
\hline \multirow[t]{2}{*}{ Model 2} & Below median & 1.49 & $(0.96,2.31)$ & 0.074 & 1.47 & $(0.68,3.19)$ & 0.332 & 1.14 & $(0.81,1.60)$ & 0.465 \\
\hline & Above median & 2.03 & $(1.28,3.22)$ & 0.003 & 1.26 & $(0.53,2.99)$ & 0.602 & 1.07 & $(0.73,1.55)$ & 0.738 \\
\hline \multirow[t]{2}{*}{ Model 3} & Below median & 1.51 & $(0.97,2.34)$ & 0.068 & 1.50 & $(0.69,3.29)$ & 0.306 & 1.11 & $(0.78,1.56)$ & 0.569 \\
\hline & Above median & 2.03 & $(1.28,3.23)$ & 0.003 & 1.28 & $(0.54,3.04)$ & 0.579 & 1.05 & $(0.72,1.53)$ & 0.787 \\
\hline \multirow[t]{2}{*}{ Model 4} & Below median & 1.47 & $(0.95,2.29)$ & 0.085 & 1.47 & $(0.67,3.21)$ & 0.333 & 1.12 & $(0.80,1.58)$ & 0.516 \\
\hline & Above median & 2.01 & $(1.26,3.18)$ & 0.003 & 1.26 & $(0.53,3.00)$ & 0.599 & 1.05 & $(0.73,1.53)$ & 0.784 \\
\hline Model 5 & Below median & 1.49 & $(0.96,2.31)$ & 0.075 & 1.47 & $(0.68,3.20)$ & 0.330 & 1.13 & $(0.81,1.59)$ & 0.466 \\
\hline & Above median & 2.01 & $(1.27,3.19)$ & 0.003 & 1.27 & $(0.54,3.02)$ & 0.584 & 1.07 & $(0.74,1.55)$ & 0.726 \\
\hline Model 6 & Below median & 1.51 & $(0.97,2.35)$ & 0.068 & 1.50 & $(0.69,3.28)$ & 0.308 & 1.11 & $(0.78,1.56)$ & 0.564 \\
\hline & Above median & 2.00 & $(1.26,3.18)$ & 0.003 & 1.28 & $(0.54,3.05)$ & 0.578 & 1.05 & $(0.73,1.53)$ & 0.780 \\
\hline
\end{tabular}


Table 3. Cont.

\begin{tabular}{|c|c|c|c|c|c|c|c|c|c|c|}
\hline & \multirow{2}{*}{ Model } & \multicolumn{3}{|c|}{ ADHD, 188 (15.9\%) } & \multicolumn{3}{|c|}{ ASD, $44(3.7 \%)$} & \multicolumn{3}{|c|}{ Other DD, $344(29.2 \%)$} \\
\hline & & Odds Ratio & $95 \% \mathrm{CI}$ & $p$-Value & Odds Ratio & $95 \% \mathrm{CI}$ & $p$-Value & Odds Ratio & $95 \% \mathrm{CI}$ & $p$-Value \\
\hline \multicolumn{11}{|c|}{ Acetaminophen burden ** } \\
\hline \multirow[t]{2}{*}{ Model 1} & Below median & 1.57 & $(1.05,2.36)$ & 0.029 & 1.85 & $(0.87,3.93)$ & 0.110 & 1.28 & $(0.92,1.76)$ & 0.139 \\
\hline & Above median & 1.88 & $(1.28,2.78)$ & 0.001 & 1.94 & $(0.92,4.08)$ & 0.080 & 1.29 & $(0.94,1.78)$ & 0.119 \\
\hline \multirow[t]{2}{*}{ Model 2} & Below median & 1.56 & $(1.01,2.42)$ & 0.045 & 1.39 & $(0.63,3.06)$ & 0.410 & 1.14 & $(0.81,1.61)$ & 0.439 \\
\hline & Above median & 1.91 & $(1.21,3.04)$ & 0.006 & 1.36 & $(0.58,3.20)$ & 0.477 & 1.05 & $(0.73,1.53)$ & 0.779 \\
\hline \multirow[t]{2}{*}{ Model 3} & Below median & 1.58 & $(1.02,2.45)$ & 0.041 & 1.43 & $(0.64,3.15)$ & 0.381 & 1.11 & $(0.79,1.57)$ & 0.543 \\
\hline & Above median & 1.92 & $(1.21,3.05)$ & 0.006 & 1.38 & $(0.59,3.25)$ & 0.457 & 1.04 & $(0.72,1.52)$ & 0.824 \\
\hline \multirow[t]{2}{*}{ Model 4} & Below median & 1.54 & $(1.00,2.39)$ & 0.052 & 1.39 & $(0.63,3.08)$ & 0.411 & 1.13 & $(0.80,1.59)$ & 0.488 \\
\hline & Above median & 1.89 & $(1.19,3.01)$ & 0.007 & 1.37 & $(0.58,3.22)$ & 0.475 & 1.04 & $(0.72,1.51)$ & 0.826 \\
\hline \multirow[t]{2}{*}{ Model 5} & Below median & 1.56 & $(1.01,2.41)$ & 0.045 & 1.39 & $(0.63,3.07)$ & 0.409 & 1.14 & $(0.81,1.61)$ & 0.442 \\
\hline & Above median & 1.90 & $(1.20,3.02)$ & 0.007 & 1.38 & $(0.59,3.25)$ & 0.459 & 1.06 & $(0.73,1.54)$ & 0.763 \\
\hline \multirow{2}{*}{ Model 6} & Below median & 1.58 & $(1.02,2.46)$ & 0.040 & 1.43 & $(0.64,3.15)$ & 0.382 & 1.11 & $(0.79,1.57)$ & 0.539 \\
\hline & Above median & 1.88 & $(1.18,3.00)$ & 0.008 & 1.39 & $(0.59,3.27)$ & 0.456 & 1.05 & $(0.72,1.52)$ & 0.815 \\
\hline
\end{tabular}

Note: NT was defined as free of any developmental disorder diagnosis; ADHD was defined as any ADHD diagnosis; ASD was defined as any ASD diagnosis without having an ADHD diagnosis; other DD was defined as any developmental disorder diagnosis other than ASD and ADHD; Model 1: Multinomial logistic regression without adjustment; Model 2: Model 1 further adjusted for maternal age at delivery, maternal race/ethnicity, maternal education, smoking before or during pregnancy, drinking before or during pregnancy, maternal BMI, parity, child's sex, delivery type, preterm birth, and birthweight; Model 3: Model 2 further adjusted for maternal fever during pregnancy; Model 4: Model 2 further adjusted for maternal intrauterine infection/inflammation during pregnancy; Model 5: Model 2 further adjusted for breastfeeding; Model 6: Model 2 further adjusted for maternal fever, maternal intrauterine infection/inflammation during pregnancy, and breastfeeding. * Inverse normal transformed intensity ** Sum of all the acetaminophen metabolites. Unchanged acetaminophen: first tertile as reference; For other exposures: no detection as reference.

We also explored if the associations between acetaminophen metabolites and ADHD varied by strata of covariables. Figure 3 presents the forest plot of the stratified analyses for binary acetaminophen burden (detected vs. non-detection) by each stratum of covariates using simple logistic regression comparing ADHD diagnosis to NT. The point estimates of the acetaminophen burden-ADHD associations were similar among strata of maternal age, smoking before or during pregnancy, maternal obesity, and early life lead exposure. On the other hand, a larger difference in the point estimate of the odds ratios was observed across strata of child's sex, parity, intrauterine infection/inflammation, alcohol drinking before or during pregnancy, delivery type, birthweight, gestational age, breastfeeding, and maternal HDL. However, tests of interaction between each covariate and binary acetaminophen burden (detected vs. non-detection) were not significant. 


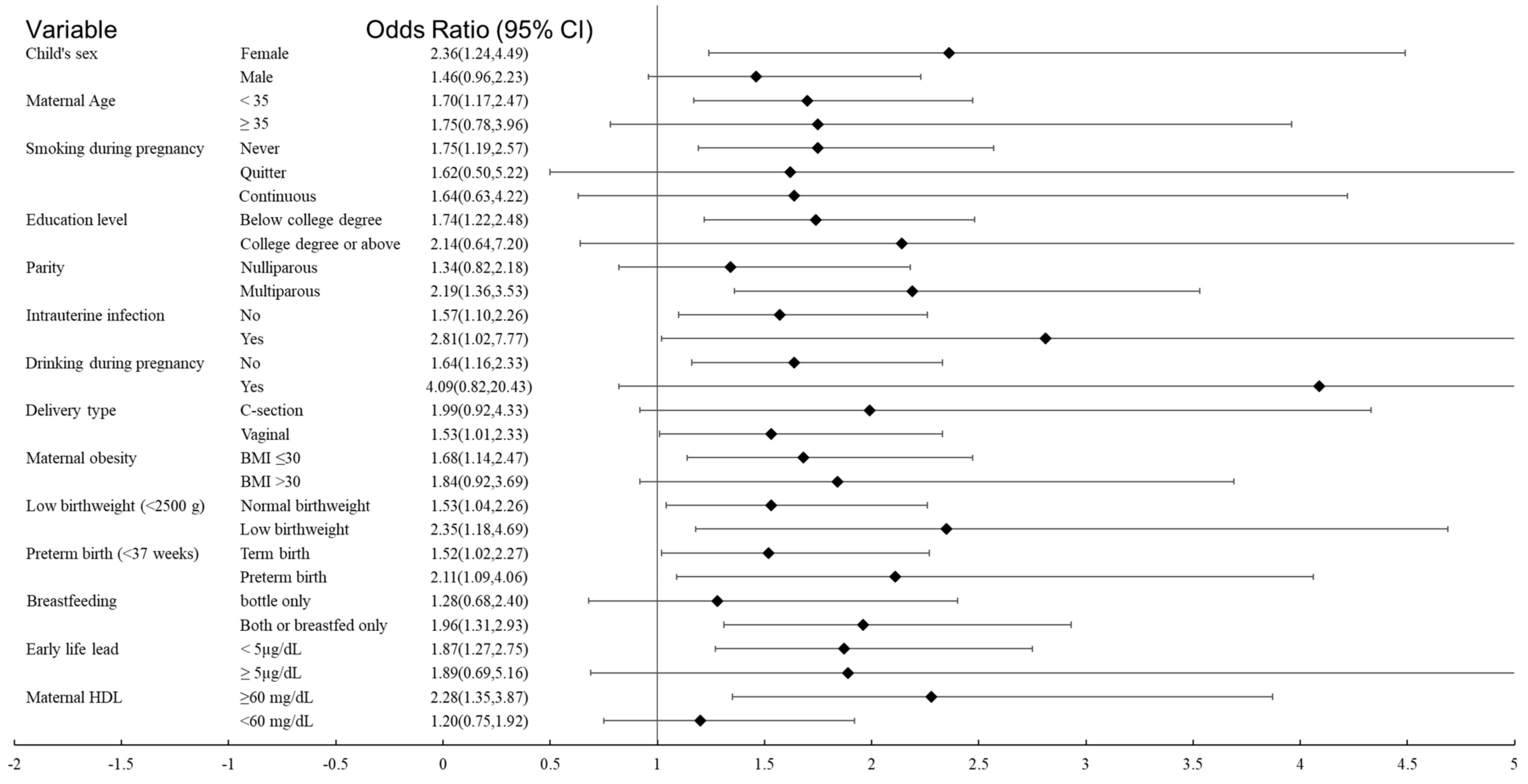

Figure 3. The forest plot for the crude association between maternal binary acetaminophen burden and the risk of ADHD diagnosis in offspring across each stratum of pertinent covariables. Acetaminophen burden is the sum of all the acetaminophen metabolites. No detection group is the reference group. 


\section{Discussion}

In this prospective birth cohort study, we found a significant positive association between maternal blood acetaminophen metabolite levels measured within 1-3 days postpartum and ADHD diagnosis in offspring; such an association was not observed for other developmental disorders. This association remained even after adjusting for indication factors of acetaminophen use (maternal fever and maternal intrauterine infection/inflammation during pregnancy) and other pertinent covariates. This study has contributed the following new information to the field.

Even though positive associations between maternal reported intake of acetaminophen during pregnancy and risk of ADHD diagnosis in their offspring have been reported by multiple independent large cohort studies [37-41], there has been a dearth of prospective birth cohort studies to examine objective biomarkers of acetaminophen use to address specific concerns about self-reported exposure and lack of dose quantification in those studies.

To our knowledge, this is the first prospective birth cohort study to examine the association between objective maternal plasma biomarkers of acetaminophen and offspring ADHD diagnosis, and to take into account a large number of potential covariables. Our study was further strengthened by the diagnosis of ADHD by both general pediatricians and developmental specialists. By demonstrating a prospective and dose-response relationship using biomarkers specific to acetaminophen intake during early postpartum, our study findings further extend the previously identified positive association between acetaminophen and ADHD from pregnancy into postpartum.

Although the causality and biological mechanisms underlying the maternal acetaminophen and child ADHD association remain to be determined, the potential for neurotoxicity is plausible according to previous findings. First, the therapeutic effect of acetaminophen inhibits prostaglandin production [33]. Prostaglandin synthesis involves multiple essential biological processes underlying the function and development of the brain, such as long-term potentiation [34], learning [35], and cerebellar development [33]. Second, accumulating studies have shown that acetaminophen not only rapidly enters the cerebrospinal fluid but also shows a profound influence on adult brain function [53-56]. Third, maternal acetaminophen can be readily transferred to the fetus through placenta [57] and to the infant via breastfeeding [58-60]. Acetaminophen remains in fetal/infant circulation much longer than it does in adults [57]. The maternal usage of phenacetin (could be converted into acetaminophen rapidly in adults) $5.5 \mathrm{~h}$ before delivery could lead to detectable acetaminophen metabolites in infant's urine until the first $26 \mathrm{~h}$ of life [57]. The prolonged detection of acetaminophen among children is due to their undeveloped liver, which slowly metabolizes the acetaminophen [61]. On the one hand, the low metabolic capacity in early life makes it safer for children to use acetaminophen because of slower production of toxic NAPQI, but, on the other hand, it makes the fetus/infant more vulnerable to maternal metabolized toxic NAPQI during pregnancy and breastfeeding. Our analysis stratified by breastfeeding history shows stronger associations among women who ever breastfeed their babies, which provides indirect support for the impact of acetaminophen through lactation. It is possible that fetal/infant exposure to maternal acetaminophen metabolites via placenta or breastfeeding, coupled with limited metabolic capacity, might lead to both direct toxic damage from maternal NAPQI and potential disruption in neurodevelopment function due to prostaglandin inhibition.

While tests of interaction were not significant (likely due to lack of power), our stratified analyses identified multiple maternal and fetal factors that may enhance the association between maternal acetaminophen metabolites and ADHD diagnosis in offspring. These potential effect modifiers are biologically plausible and warrant further investigations. For instance, we found that the effect size of acetaminophen use on the risk of ADHD diagnosis is more pronounced among women who drank alcohol six months before or during pregnancy. Effect modification by alcohol is supported by biological studies $[62,63]$. A mechanistic study showed that ethanol could cause induction of cytochrome P450 2E1 and selective depletion of mitochondrial glutathione, which could lead to limited clearance capacity of the toxic NAPQI [63]. Additionally, the stronger and more significant 
acetaminophen-ADHD association among female children indicates the need to further investigate the potential sex-specific biological mechanism underlying the acetaminophen exposure.

Our study also had some limitations. First, this study only included a one-time measurement of maternal acetaminophen metabolite levels within 1-3 days postpartum. Acetaminophen has a 2-3 h half-life in adults [64], thus the detectable levels of acetaminophen biomarkers can only reflect women's recent use, which could be influenced by both dosage and metabolism capacity. As a result, we cannot pinpoint the exact dosage and usage pattern at specific time periods (pre-conception, specific trimester during pregnancy, perinatal and postnatal period) based on this one-time measurement. Nonetheless, women with detectable levels of acetaminophen biomarkers are likely to be more regular users. The findings would be strengthened if we could have included maternal acetaminophen metabolite measures taken at least once for each trimester as well as cord blood biomarkers. Given the fact that the rate of prenatal acetaminophen use during pregnancy is over $65 \%$ in the U.S. [32], the one-time measurement in our study likely reflects maternal acetaminophen use during the peripartum period. Our future study with cord blood metabolite measurements will provide further confirmation. Second, our metabolite measurement method did not include acetaminophen sulfate, which accounts for $30-44 \%$ of the total metabolites of acetaminophen under the normal dosage [48]. Fortunately, the total acetaminophen burden can be estimated based on the relatively stable proportions of other measured acetaminophen metabolites [48]. Third, although we adjusted for major known risk factors of ADHD and indication of acetaminophen use, we could not adjust for several familial factors identified in previous studies [3-5,13-15,65]. We also cannot rule out the possibility of unmeasured or unknown residual confounding, although our propensity score analyses seek to adjust for such confounding and provide additional credibility for our findings. Lastly, our study sample consists of a predominantly urban low-income minority population. This characteristic may limit the generalization of our results to all pregnant women living in the U.S.

\section{Conclusions}

Maternal plasma biomarkers of acetaminophen use measured within a few days after delivery were specifically associated with increased risk of ADHD diagnosis in offspring, not with other developmental disorders. This association remained after adjusting for multiple previously identified potential confounders and potential indications for acetaminophen use. While our study provides the first objective biomarker evidence of the relationship between maternal acetaminophen use and ADHD diagnosis in offspring, we could not provide definitive support for a causal inference of this relationship, given the observational nature of this study and the limitations outlined above. However, our evidence drawn from the perinatal period lends further support for the association between maternal acetaminophen use and ADHD in offspring, which has been illustrated by multiple large studies of acetaminophen use during pregnancy via self-reported information. If further confirmed, these results may help address concerns raised by the Society for Maternal-Fetal Medicine and the U.S. Food and Drug Administration [42,43]. Taking past findings together with the findings from this study, the potential adverse effect of maternal acetaminophen use on ADHD risk in offspring warrants additional investigations.

Supplementary Materials: The following are available online at http:/ /www.mdpi.com/2076-3425/8/7/127/s1, Figure S1: Flowchart of sample included in the analysis; Table S1: Maternal and child characteristics for participants excluded and included in the analysis; Table S2: List of ICD-9 and ICD-10 codes for the diagnosis of each developmental disorder; Table S3: The association between maternal acetaminophen metabolites and the risk of ADHD diagnosis only, ASD diagnosis only, and diagnoses of both ADHD and ASD in offspring; Table S4: The association between maternal acetaminophen metabolites and the risk of ADHD diagnosis, ASD diagnosis (excluding ADHD), and other DD diagnosis in offspring using propensity score weighted multinomial logistic regression models.

Author Contributions: Y.J. conceptualized the study; assumed primary responsibility for laboratory analysis, data management, and statistical analyses; and drafted and revised this manuscript. X.H., G.W., J.T., and C.P. participated in data collection, data management, and critically reviewed and revised the manuscript. A.W.R. and 
T.R.B. participated in drafting and revising this manuscript. L.-C.L., H.-J.T., N.T.M., H.J., I.B., M.D.F. and A.P. provided critical review and input on the study design, data analyses, interpretation of data, and participated in the revision of the manuscript. X.W. is the founder and principal investigator of the Boston Birth Cohort; she oversaw participant recruitment, follow-up and data collection, conceptualized the study and provided critical inputs on the study design, data analyses, interpretation of data, initial draft and revision of the manuscript.

Funding: This work was supported by the Health Resources and Services Administration (HRSA) of the U.S. Department of Health and Human Services (HHS) under grant number R40MC27443 and UJ2MC31074. The Boston Birth Cohort (the parent study) is supported in part by the March of Dimes PERI grants (20-FY02-56 and 21-FY07-605); and the National Institutes of Health (NIH) grants (R21ES011666, R01HD041702, R21HD066471, U01AI090727, R21AI079872, 2R01HD041702, and R01HD086013). The content and conclusions contained in this article are those of the authors and should not be construed as the official position or policy of, nor should any endorsements be inferred by HRSA, HHS or the U.S. Government.

Conflicts of Interest: The authors declare no conflict of interest.

\section{References}

1. Pastor, P.N.; Duran, C.; Reuben, C. Quickstats: Percentage of children and adolescents aged 5-17 years with diagnosed attention-deficit/hyperactivity disorder (adhd), by race and hispanic ethnicity-National health interview survey, United States, 1997-2014. Morb. Mortal. Wkly. Rep. 2015, 64, 925.

2. Danielson, M.L.; Bitsko, R.H.; Ghandour, R.M.; Holbrook, J.R.; Kogan, M.D.; Blumberg, S.J. Prevalence of parent-reported adhd diagnosis and associated treatment among U.S. Children and adolescents, 2016. J. Clin. Child Adolesc. Psychol. 2018, 47, 199-212. [CrossRef] [PubMed]

3. Patterson, G.R.; DeGarmo, D.; Forgatch, M.S. Systematic changes in families following prevention trials. J. Abnorm. Child Psychol. 2004, 32, 621-633. [CrossRef] [PubMed]

4. Scott, S.; O'Connor, T.G.; Futh, A.; Matias, C.; Price, J.; Doolan, M. Impact of a parenting program in a high-risk, multi-ethnic community: The pals trial. J. Child Psychol. Psychiatry 2010, 51, 1331-1341. [CrossRef] [PubMed]

5. Jaffee, S.R.; Caspi, A.; Moffitt, T.E.; Polo-Tomas, M.; Price, T.S.; Taylor, A. The limits of child effects: Evidence for genetically mediated child effects on corporal punishment but not on physical maltreatment. Dev. Psychol. 2004, 40, 1047-1058. [CrossRef] [PubMed]

6. Costello, E.J.; Compton, S.N.; Keeler, G.; Angold, A. Relationships between poverty and psychopathology: A natural experiment. JAMA 2003, 290, 2023-2029. [CrossRef] [PubMed]

7. Langley, K.; Rice, F.; van den Bree, M.B.; Thapar, A. Maternal smoking during pregnancy as an environmental risk factor for attention deficit hyperactivity disorder behaviour. A review. Minerva Pediatr. 2005, 57, 359-371. [PubMed]

8. $\quad$ Linnet, K.M.; Dalsgaard, S.; Obel, C.; Wisborg, K.; Henriksen, T.B.; Rodriguez, A.; Kotimaa, A.; Moilanen, I.; Thomsen, P.H.; Olsen, J.; et al. Maternal lifestyle factors in pregnancy risk of attention deficit hyperactivity disorder and associated behaviors: Review of the current evidence. Am. J. Psychiatry 2003, 160, 1028-1040. [CrossRef] [PubMed]

9. Glover, V. Annual research review: Prenatal stress and the origins of psychopathology: An evolutionary perspective. J. Child Psychol. Psychiatry 2011, 52, 356-367. [CrossRef] [PubMed]

10. Grizenko, N.; Shayan, Y.R.; Polotskaia, A.; Ter-Stepanian, M.; Joober, R. Relation of maternal stress during pregnancy to symptom severity and response to treatment in children with adhd. J. Psychiatry Neurosci. 2008, 33, 10-16. [PubMed]

11. Pheula, G.F.; Rohde, L.A.; Schmitz, M. Are family variables associated with adhd, inattentive type? A case-control study in schools. Eur. Child Adolesc. Psychiatry 2011, 20, 137-145. [CrossRef] [PubMed]

12. Scahill, L.; Schwab-Stone, M.; Merikangas, K.R.; Leckman, J.F.; Zhang, H.; Kasl, S. Psychosocial and clinical correlates of adhd in a community sample of school-age children. J. Am. Acad. Child Adolesc. Psychiatry 1999, 38, 976-984. [CrossRef] [PubMed]

13. Lifford, K.J.; Harold, G.T.; Thapar, A. Parent-child relationships and adhd symptoms: A longitudinal analysis. J. Abnorm. Child Psychol. 2008, 36, 285-296. [CrossRef] [PubMed]

14. O'Connor, T.G.; Rutter, M. Attachment disorder behavior following early severe deprivation: Extension and longitudinal follow-up. English and Romanian adoptees study team. J. Am. Acad. Child Adolesc. Psychiatry 2000, 39, 703-712. [CrossRef] [PubMed] 
15. Rutter, M.; Beckett, C.; Castle, J.; Colvert, E.; Kreppner, J.; Mehta, M.; Stevens, S.; Sonuga-Barke, E. Effects of profound early institutional deprivation: An overview of findings from a uk longitudinal study of romanian adoptees. Eur. J. Dev. Psychol. 2007, 4, 332-350. [CrossRef]

16. Rodriguez, A.; Miettunen, J.; Henriksen, T.B.; Olsen, J.; Obel, C.; Taanila, A.; Ebeling, H.; Linnet, K.M.; Moilanen, I.; Jarvelin, M.R. Maternal adiposity prior to pregnancy is associated with adhd symptoms in offspring: Evidence from three prospective pregnancy cohorts. Int. J. Obes. 2008, 32, 550-557. [CrossRef] [PubMed]

17. Chen, Q.; Sjolander, A.; Langstrom, N.; Rodriguez, A.; Serlachius, E.; D'Onofrio, B.M.; Lichtenstein, P.; Larsson, H. Maternal pre-pregnancy body mass index and offspring attention deficit hyperactivity disorder: A population-based cohort study using a sibling-comparison design. Int. J. Epidemiol. 2014, 43, 83-90. [CrossRef] [PubMed]

18. Sengupta, S.M.; Fortier, M.E.; Thakur, G.A.; Bhat, V.; Grizenko, N.; Joober, R. Parental psychopathology in families of children with attention-deficit/hyperactivity disorder and exposed to maternal smoking during pregnancy. J. Child Psychol. Psychiatry 2015, 56, 122-129. [CrossRef] [PubMed]

19. Gard, A.M.; Owens, E.B.; Hinshaw, S.P. Prenatal smoke exposure predicts hyperactive/impulsive but not inattentive adhd symptoms in adolescent and young adult girls. Infant. Child Dev. 2016, 25, 339-351. [CrossRef] [PubMed]

20. Gustavson, K.; Ystrom, E.; Stoltenberg, C.; Susser, E.; Suren, P.; Magnus, P.; Knudsen, G.P.; Smith, G.D.; Langley, K.; Rutter, M.; et al. Smoking in pregnancy and child adhd. Pediatrics 2017, 139. [CrossRef] [PubMed]

21. Eilertsen, E.M.; Gjerde, L.C.; Reichborn-Kjennerud, T.; Orstavik, R.E.; Knudsen, G.P.; Stoltenberg, C.; Czajkowski, N.; Roysamb, E.; Kendler, K.S.; Ystrom, E. Maternal alcohol use during pregnancy and offspring attention-deficit hyperactivity disorder (adhd): A prospective sibling control study. Int. J. Epidemiol. 2017, 46, 1633-1640. [CrossRef] [PubMed]

22. Bhutta, A.T.; Cleves, M.A.; Casey, P.H.; Cradock, M.M.; Anand, K.J. Cognitive and behavioral outcomes of school-aged children who were born preterm: A meta-analysis. JAMA 2002, 288, 728-737. [CrossRef] [PubMed]

23. Bouchard, M.F.; Bellinger, D.C.; Wright, R.O.; Weisskopf, M.G. Attention-deficit/hyperactivity disorder and urinary metabolites of organophosphate pesticides. Pediatrics 2010, 125, e1270-e1277. [CrossRef] [PubMed]

24. Eubig, P.A.; Aguiar, A.; Schantz, S.L. Lead and pcbs as risk factors for attention deficit/hyperactivity disorder. Environ. Health Perspect. 2010, 118, 1654-1667. [CrossRef] [PubMed]

25. Sagiv, S.K.; Thurston, S.W.; Bellinger, D.C.; Tolbert, P.E.; Altshul, L.M.; Korrick, S.A. Prenatal organochlorine exposure and behaviors associated with attention deficit hyperactivity disorder in school-aged children. Am. J. Epidemiol. 2010, 171, 593-601. [CrossRef] [PubMed]

26. Braun, J.M.; Kahn, R.S.; Froehlich, T.; Auinger, P.; Lanphear, B.P. Exposures to environmental toxicants and attention deficit hyperactivity disorder in U.S. Children. Environ. Health Perspect. 2006, 114, 1904-1909. [PubMed]

27. Froehlich, T.E.; Lanphear, B.P.; Auinger, P.; Hornung, R.; Epstein, J.N.; Braun, J.; Kahn, R.S. Association of tobacco and lead exposures with attention-deficit/hyperactivity disorder. Pediatrics 2009, 124, e1054-e1063. [CrossRef] [PubMed]

28. Nigg, J.T.; Nikolas, M.; Mark Knottnerus, G.; Cavanagh, K.; Friderici, K. Confirmation and extension of association of blood lead with attention-deficit/hyperactivity disorder (adhd) and adhd symptom domains at population-typical exposure levels. J. Child Psychol. Psychiatry 2010, 51, 58-65. [CrossRef] [PubMed]

29. Ji, Y.; Hong, X.; Wang, G.; Chatterjee, N.; Riley, A.W.; Lee, L.C.; Surkan, P.J.; Bartell, T.R.; Zuckerman, B.; Wang, X. A prospective birth cohort study on early childhood lead levels and attention deficit hyperactivity disorder: New insight on sex differences. J. Pediatr. 2018. [CrossRef] [PubMed]

30. Wolraich, M.L. An association between prenatal acetaminophen use and adhd: The benefits of large data sets. Pediatrics 2017, 140. [CrossRef] [PubMed]

31. Lupattelli, A.; Spigset, O.; Twigg, M.J.; Zagorodnikova, K.; Mardby, A.C.; Moretti, M.E.; Drozd, M.; Panchaud, A.; Hameen-Anttila, K.; Rieutord, A.; et al. Medication use in pregnancy: A cross-sectional, multinational web-based study. BMJ Open 2014, 4, e004365. [CrossRef] [PubMed]

32. Werler, M.M.; Mitchell, A.A.; Hernandez-Diaz, S.; Honein, M.A. Use of over-the-counter medications during pregnancy. Am. J. Obstet. Gynecol. 2005, 193, 771-777. [CrossRef] [PubMed] 
33. Dean, S.L.; Knutson, J.F.; Krebs-Kraft, D.L.; McCarthy, M.M. Prostaglandin e2 is an endogenous modulator of cerebellar development and complex behavior during a sensitive postnatal period. Eur. J. Neurosci. 2012, 35, 1218-1229. [CrossRef] [PubMed]

34. Yamagata, K.; Andreasson, K.I.; Kaufmann, W.E.; Barnes, C.A.; Worley, P.F. Expression of a mitogen-inducible cyclooxygenase in brain neurons: Regulation by synaptic activity and glucocorticoids. Neuron 1993, 11, 371-386. [CrossRef]

35. Shaw, K.N.; Commins, S.; O'Mara, S.M. Deficits in spatial learning and synaptic plasticity induced by the rapid and competitive broad-spectrum cyclooxygenase inhibitor ibuprofen are reversed by increasing endogenous brain-derived neurotrophic factor. Eur. J. Neurosci. 2003, 17, 2438-2446. [CrossRef] [PubMed]

36. Olsen, J.; Liew, Z. Fetal programming of mental health by acetaminophen? Response to the smfm statement: Prenatal acetaminophen use and adhd. Expert Opin. Drug Saf. 2017, 16, 1395-1398. [CrossRef] [PubMed]

37. Ystrom, E.; Gustavson, K.; Brandlistuen, R.E.; Knudsen, G.P.; Magnus, P.; Susser, E.; Davey Smith, G.; Stoltenberg, C.; Suren, P.; Haberg, S.E.; et al. Prenatal exposure to acetaminophen and risk of adhd. Pediatrics 2017, 140. [CrossRef] [PubMed]

38. Liew, Z.; Ritz, B.; Rebordosa, C.; Lee, P.C.; Olsen, J. Acetaminophen use during pregnancy, behavioral problems, and hyperkinetic disorders. JAMA Pediatr. 2014, 168, 313-320. [CrossRef] [PubMed]

39. Thompson, J.M.; Waldie, K.E.; Wall, C.R.; Murphy, R.; Mitchell, E.A. ABC Study Group. Associations between acetaminophen use during pregnancy and adhd symptoms measured at ages 7 and 11 years. PLOS ONE 2014, 9, e108210. [CrossRef] [PubMed]

40. Stergiakouli, E.; Thapar, A.; Davey Smith, G. Association of acetaminophen use during pregnancy with behavioral problems in childhood: Evidence against confounding. JAMA Pediatr. 2016, 170, 964-970. [CrossRef] [PubMed]

41. Avella-Garcia, C.B.; Julvez, J.; Fortuny, J.; Rebordosa, C.; Garcia-Esteban, R.; Galan, I.R.; Tardon, A.; Rodriguez-Bernal, C.L.; Iniguez, C.; Andiarena, A.; et al. Acetaminophen use in pregnancy and neurodevelopment: Attention function and autism spectrum symptoms. Int. J. Epidemiol. 2016, 45, 1987-1996. [CrossRef] [PubMed]

42. Society for Maternal-Fetal Medicine Publications Committee. Prenatal acetaminophen use and outcomes in children. Am. J. Obstet. Gynecol. 2017, 216, B14-B15.

43. Food and Drug Administration. Fda Has Reviewed Possible Risks of Pain Medication during Pregnancy. Available online: https:/ / www.fda.gov/Drugs/DrugSafety/ucm429117.htm (accessed on 7 January 2018).

44. Wang, G.; Divall, S.; Radovick, S.; Paige, D.; Ning, Y.; Chen, Z.; Ji, Y.; Hong, X.; Walker, S.O.; Caruso, D.; et al. Preterm birth and random plasma insulin levels at birth and in early childhood. JAMA 2014, 311, 587-596. [CrossRef] [PubMed]

45. Wang, X.; Zuckerman, B.; Pearson, C.; Kaufman, G.; Chen, C.; Wang, G.; Niu, T.; Wise, P.H.; Bauchner, H.; $\mathrm{Xu}, \mathrm{X}$. Maternal cigarette smoking, metabolic gene polymorphism, and infant birth weight. JAMA 2002, 287, 195-202. [CrossRef] [PubMed]

46. Li, M.; Fallin, M.D.; Riley, A.; Landa, R.; Walker, S.O.; Silverstein, M.; Caruso, D.; Pearson, C.; Kiang, S.; Dahm, J.L.; et al. The association of maternal obesity and diabetes with autism and other developmental disabilities. Pediatrics 2016, 137, e20152206. [CrossRef] [PubMed]

47. Kumar, R.; Tsai, H.J.; Hong, X.; Liu, X.; Wang, G.; Pearson, C.; Ortiz, K.; Fu, M.; Pongracic, J.A.; Bauchner, H.; et al. Race, ancestry, and development of food-allergen sensitization in early childhood. Pediatrics 2011, 128, e821-e829. [CrossRef] [PubMed]

48. Mazaleuskaya, L.L.; Sangkuhl, K.; Thorn, C.F.; FitzGerald, G.A.; Altman, R.B.; Klein, T.E. Pharmgkb summary. Pharmacogenet. Genom. 2015, 25, 416-426. [CrossRef] [PubMed]

49. Centers for Disease Control and Prevention. CDC Response to Advisory Committee on Childhood Lead Poisoning Prevention Recommendations in "Low Level Lead Exposure Harms Children: A Renewed Call of Primary Prevention"; Centers for Disease Control and Prevention: Atlanta, GA, USA, 2012.

50. Ji, Y.; Riley, A.W.; Lee, L.C.; Volk, H.; Hong, X.; Wang, G.; Angomas, R.; Stivers, T.; Wahl, A.; Ji, H.; et al. A prospective birth cohort study on maternal cholesterol levels and offspring attention deficit hyperactivity disorder: New insight on sex differences. Brain Sci. 2017, 8, 3. [CrossRef] [PubMed]

51. Menard, S. Applied Logistic Regression Analysis; Sage: Newcastle upon Tyne, UK, 2002; Volume 106.

52. Goodlad, J.K.; Marcus, D.K.; Fulton, J.J. Lead and attention-deficit/hyperactivity disorder (adhd) symptoms: A meta-analysis. Clin. Psychol. Rev. 2013, 33, 417-425. [CrossRef] [PubMed] 
53. Randles, D.; Heine, S.J.; Santos, N. The common pain of surrealism and death: Acetaminophen reduces compensatory affirmation following meaning threats. Psychol. Sci. 2013, 24, 966-973. [CrossRef] [PubMed]

54. Durso, G.R.; Luttrell, A.; Way, B.M. Over-the-counter relief from pains and pleasures alike: Acetaminophen blunts evaluation sensitivity to both negative and positive stimuli. Psychol. Sci. 2015, 26, 750-758. [CrossRef] [PubMed]

55. Dewall, C.N.; Macdonald, G.; Webster, G.D.; Masten, C.L.; Baumeister, R.F.; Powell, C.; Combs, D.; Schurtz, D.R.; Stillman, T.F.; Tice, D.M.; et al. Acetaminophen reduces social pain: Behavioral and neural evidence. Psychol. Sci. 2010, 21, 931-937. [CrossRef] [PubMed]

56. Randles, D.; Kam, J.W.; Heine, S.J.; Inzlicht, M.; Handy, T.C. Acetaminophen attenuates error evaluation in cortex. Soc. Cogn. Affect. Neurosci. 2016, 11, 899-906. [CrossRef] [PubMed]

57. Levy, G.; Garrettson, L.K.; Soda, D.M. Letter: Evidence of placental transfer of acetaminophen. Pediatrics 1975, 55, 895. [CrossRef] [PubMed]

58. Berlin, C.M., Jr.; Yaffe, S.J.; Ragni, M. Disposition of acetaminophen in milk, saliva, and plasma of lactating women. Pediatr. Pharmacol. 1980, 1, 135-141.

59. Matheson, I.; Lunde, P.K.; Notarianni, L. Infant rash caused by paracetamol in breast milk? Pediatrics 1985, 76, 651-652. [PubMed]

60. Notarianni, L.J.; Oldham, H.G.; Bennett, P.N. Passage of paracetamol into breast milk and its subsequent metabolism by the neonate. Br. J. Clin. Pharmacol. 1987, 24, 63-67. [CrossRef] [PubMed]

61. Penna, A.; Buchanan, N. Paracetamol poisoning in children and hepatotoxicity. Br. J. Clin. Pharmacol. 1991, 32, 143-149. [CrossRef] [PubMed]

62. McClain, C.J.; Kromhout, J.P.; Peterson, F.J.; Holtzman, J.L. Potentiation of acetaminophen hepatotoxicity by alcohol. JAMA 1980, 244, 251-253. [CrossRef] [PubMed]

63. Zhao, P.; Kalhorn, T.F.; Slattery, J.T. Selective mitochondrial glutathione depletion by ethanol enhances acetaminophen toxicity in rat liver. Hepatology 2002, 36, 326-335. [CrossRef] [PubMed]

64. Burke, A.; Smyth, E.; FitzGerald, G.A. Analgesic-antipyretic agents; pharmacotherapy of gout. Pharmacol. Basis Ther. 2006, 1, 671-716.

65. Ystrom, E.; Vollrath, M.E.; Nordeng, H. Effects of personality on use of medications, alcohol, and cigarettes during pregnancy. Eur. J. Clin. Pharmacol. 2012, 68, 845-851. [CrossRef] [PubMed] 